\title{
Intrathecal cell therapy with autologous stromal cells increases cerebral glucose metabolism and can offer a new approach to the treatment of Alzheimer's type dementia
}

\author{
JESÚS VAQUERO ${ }^{1}$, MERCEDES ZURITA ${ }^{1}$, JORGE MUCIENTES ${ }^{2}$, MARIA L. PASCUAL $^{3}$, \\ CECILIA FERNÁNDEZ-MATEOS ${ }^{1}$, ESTEFANIA GARCIA ${ }^{4}$ \& SARA FERNÁNDEZ-GUINEA $^{4}$ \\ ${ }^{1}$ Neurosurgery Service, Puerta de Hierro-Majadahonda Hospital, Autonomous University, Madrid, Spain, ${ }^{2}$ Nuclear \\ Medicine Service, Puerta de Hierro-Majadahonda Hospital, Autonomous University, Madrid, Spain, ${ }^{3}$ Neurology \\ Service, Puerta de Hierro-Majadahonda Hospital, Autonomous University, Madrid, Spain, and ${ }^{4}$ Department of \\ Experimental Psychology, Faculty of Psychology, Complutense University, Madrid, Spain
}

\begin{abstract}
Background aims: After recent observations that intrathecal administration of autologous bone marrow mesenchymal stromal cells (MSCs) increases cerebral metabolism in patients with severe traumatic brain injury (TBI), we examined this type of cell therapy in Alzheimer's type dementia. Methods: Three patients with clinical diagnosis of Alzheimer's disease received every 3 months 100 million autologous MSCs by intrathecal route, until a total dose of 300 million. Results: During cell therapy the patients showed arrest in neurological deterioration and two of them manifested clear improvement of previous symptoms. A global increase in cerebral glucose metabolism, measured using $18 \mathrm{~F}$-fluorodeoxyglucose positron emission tomography (18F-FDG-PET), was observed after every administration of cell therapy. Conclusions: Our present findings suggest that intrathecal administrations of autologous MSCs can be a new strategy for the treatment of Alzheimer's dementia.
\end{abstract}

Key Words: Alzheimer's disease, brain metabolism, cell therapy, dementia

\section{Introduction}

Alzheimer's disease (AD) and related dementias represent fatal neurodegenerative disorders for which at present we do not have an effective treatment. In these patients, a decrease in brain glucose metabolism is considered a crucial event related to cognitive impairment [1-3]. For this reason, generally the medications used in $\mathrm{AD}$ pursue an increase in brain glucose metabolism [4-6].

Recently we reported that intrathecal administration of bone marrow mesenchymal stromal cells (MSCs) in patients suffering from neurological dysfunction after severe traumatic brain injury (TBI) can achieve a progressive increase in brain glucose metabolism, measured using $18 \mathrm{~F}$-fluorodeoxyglucose positron emission tomography (18F-FDG-PET), and it can be associated with neurocognitive improvement [7]. After these findings, we speculate that this type of cell therapy could also be useful in $\mathrm{AD}$ and Alzheimer's type dementias (ATD).

\section{Methods}

The Spanish Agency for Medicaments and Health Products (EAMPS) and the Medical Management of Puerta de Hierro-Majadahonda Hospital authorized the intrathecal administration (into subarachnoid space, through lumbar puncture) of our NC1 medicament in three patients with diagnosis of $\mathrm{AD}$. Ethical aspects and possible benefits were considered. The study was conducted in accordance with the principles of the Declaration of Helsinki [8] and good clinical practice guidelines [9]. Informed consent was obtained, after explaining the experimental nature of the treatment and the previous experience obtained with intrathecal administration of our NC1 medicament in patients with neurological sequelae due to spinal cord injury (SCI) [10].

$\mathrm{NC} 1$ is a cell therapy medicament developed in our cleanroom of the neurosurgical service of the Puerta de Hierro-Majadahonda Hospital, and is currently approved as a medicament under clinical 
investigation (Producto en investigación (PEI) number 12-141). It consists of expanded autologous MSCs obtained from bone marrow and autologous plasma as its excipient. Techniques for culture and expansion of MSCs, phenotypic characterization, formulation and packaging have been previously described $[10,11]$ and are summarized in the Supplementary Appendix. In our medicament, MSCs have a viability always $\geq 95 \%$ as established by our quality criteria, and this viability remains constant for at least $10 \mathrm{~h}$. The administration of the cells through a lumbar puncture is a procedure that only lasts about $10 \mathrm{~min}$. Therefore, cell viability remains the same after cell transplantation.

Clinical and neuroimage studies were performed on our patients. Cerebral beta-amyloid neuritic plaques were detected with $18 \mathrm{~F}$-Flutemetamol positron emission tomography, and brain glucose metabolism was studied using 18 F-FDG-PET. Neuropsychological studies included Mini Mental State Examination (MMSE) scale [12], Rey Complex Figure Test (CRFT) [13], Stroop test [14], Spanish adaptation to California Verbal Learning Test (CVLT) [15] and Wechler Adult Intelligence Scale (WAIS-III) [16]. Statistical study of neuropsychological scores was performed using the non-overlap of all pairs (NAP) test [17].

\section{Report of cases}

\section{Case 1}

A 65-year-old man, with more than 20 years of formal education and family history of $\mathrm{AD}$, was diagnosed with $\mathrm{AD} 2$ years before coming to us to evaluate for cell therapy treatment. At this moment, he described a slow but progressive neurological deterioration. In July 2017, he stopped taking his medication (donepezil, $5 \mathrm{mg} / \mathrm{d}$ ). Cell therapy was administered between November 2017 and May 2018 (100 million MSCs every 3 months). At the end of treatment, the patient and his family considered that he was stable, with no progression, but without clear improvement of the symptoms previous to cell therapy. A neuropsychological assessment was performed before the treatment (pre) and 2 weeks after each MSC dose (post 1, post 2 and post 3). The results showed a slight improvement in MMSE [12]: pre, 27 points; post 1, 25; post 2, 28; and post 3, 27; NAP = small effect. Short-term visual memory [13] reached 12 points at the end of cell therapy (post 3), being 9.5 points previous, 8 points at post 1 and 8.5 points at post 2 . However, there were statistically significant improvements on longterm visual memory [13]: pre, 6.5 points; post 1,6 points; post 2, 8.5 points; and post 3, 8.5 points; $\mathrm{NAP}=$ medium effect. Mental processing speed [16] showed the following: pre, 33 points; post 1, 38 points; post 2, 39 points; and post 3, 37 points; $\mathrm{NAP}=$ strong effect. Inhibition of automatic response [14] showed the following: pre, 49 points; post 1,48 points; post 2,57 points; and post 3,54 points; $\mathrm{NAP}=$ medium effect.

Studies with 18F-FDG-PET showed progressive increases in global brain glucose metabolism during cell therapy (Figure 1).

\section{Case 2}

A 56-year-old man, with 20 years of formal education and no familiy history of dementia, was diagnosed with $\mathrm{AD} 2$ years before coming to us to evaluate for cell therapy treatment. The patient had not accepted medication for his cognitive problems, except vitamin supplements. He related progressive difficulties in his work, showing problems remembering how to organize simple activities or manage easy tasks on his computer. He also had exhibited several spatial disorientation episodes. He received intrathecal cell therapy between November 2017 and June 2018 (100 million MSCs every 3 months). In July 2018, after finishing cell therapy, the patient

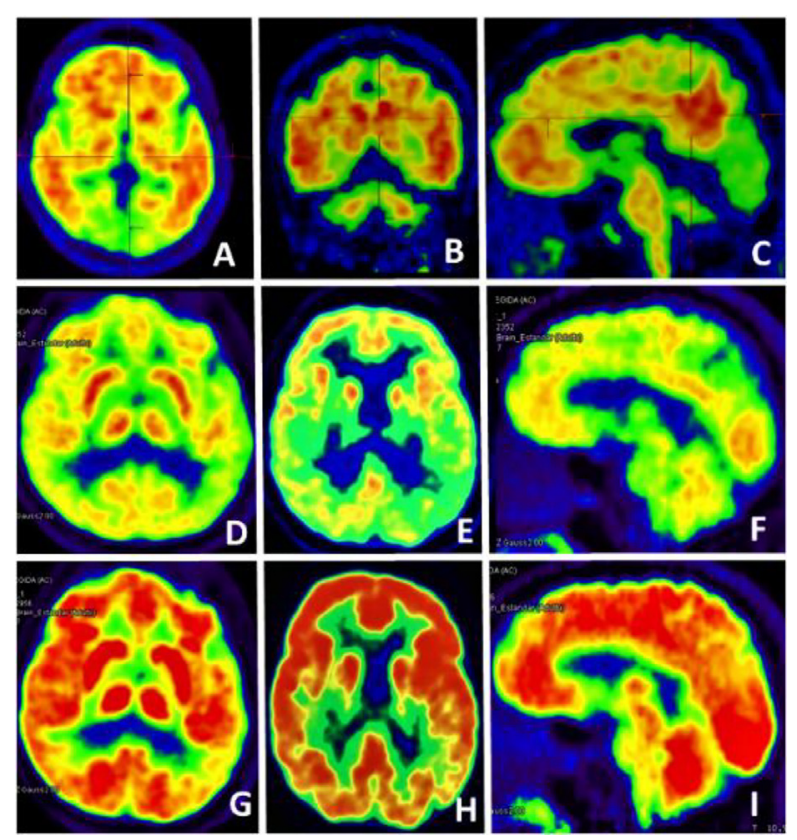

Figure 1. PET studies in Case 1. (A-C) Detection of betaamyloid neuritic plaques with $18 \mathrm{~F}-$ Flutemetamol-PET. (D-F) 18 F-FDG-PET prior to cell therapy. This study showed hypometabolism in the left parietotemporal territory, with involvement of the precuneus and posterior cingulate. A decrease in glucose uptake in the mesial region and anterior poles of both temporal lobes with hippocampal territory affectation and a focal hypometabolism on the right lateral temporal territory were also observed. (G-I) 18F-FDG-PET 1 week after the first intrathecal administration of 100 million MSCs, showing a strong increase in cerebral glucose metabolism. 
mentioned an appreciable improvement in his daily activities, especially using his computer and with no other disorientation episodes. A neuropsychological assessment was performed before the treatment (pre) and 2 weeks after every MSC dose (post 1, post 2 and post 3). Neuropsychological examination showed improvement in MMSE [12] (28 points, 27 points, 27 points and 30 points at prior, post 1 , post 2 and after cell therapy, respectively; NAP = small effect). Verbal learning [15] showed the following: pre, 38 points; post 1,59 points; post 2,54 points; and post 3, 59 points; NAP = strong effect. Verbal short-term memory test [15] showed the following: pre, 12 points; post 1,14 points; post 2,14 points; and post 3,14 points; NAP = strong effect. Verbal long-term memory test [15] showed the following: pre, 10 points; post 1,13 points; post 2,13 points; and post 3, 14 points; NAP = strong effect. Attention [16] showed the following: pre, 12 points; post 1,13 points; post 2, 16 points; and post 3, 15 points; NAP $=$ strong effect. Mental processing speed [16] showed the following: pre, 56 points; post 1, 59 points; post 2, 73 points; and post 3, 72 points; $\mathrm{NAP}=$ strong effect. Study of the inhibition of automatic response [14] showed the following: pre, 50 points; post 1, 46 points; post 2, 49 points; and post 3, 57 points; $\mathrm{NAP}=$ small effect.

$18 \mathrm{~F}-\mathrm{FDG}-\mathrm{PET}$ showed progressive increases in global brain glucose metabolism during cell therapy (Figure 2).

\section{Case 3}

Our case 3 was a 63-year-old man, with more than 20 years of formal education and maternal familiy history of $\mathrm{AD}$. He was diagnosed at the age of
61 years with dementia due to basal frontotemporal atrophy. After demonstration of intracerebral betaamyloid neuritic plaques using $18 \mathrm{~F}-$ FlutemetamolPET, a diagnosis of $\mathrm{AD}$ was made. He presented important memory problems and disorientation episodes, which had caused him to stop working. He had rejected donopezil. Cell therapy was administered between November 2016 and May 2017 (100 million MSCs every 3 months). After first administration, the patient experienced an amelioration, especially in orientation, corroborated by his family. The benefit was progressive, and at the end of the treatment, the patient could manage his fiscal obligations and going out without any disorientation problems. The family also reported better communication skills and evident greater participation in interactions with family or friends. The patient showed a score of 30 on MMSE [12] prior to the treatment and maintained it at the end of treatment.

Neuropsychological assessment was performed before the treatment (pre) and 2 weeks after each MSCs administration (post 1, post 2 and post 3 ). The data showed a statistically significant improvement [17] in verbal learning [15]: pre, 28 points; post 1,32 points; post 2, 36 points; and post 3, 40 points; NAP = strong effect. Verbal short-term memory [15] showed the following: pre, 5 points; post 1 , 6 points; post 2, 5 points; and post 3, 7 points; $\mathrm{NAP}=$ medium effect. Verbal long-term memory [15] showed the following: pre, 4 points; post 1,8 points; post 2,5 points; and post 3,8 points; NAP $=$ strong effect. The study of semantic memory [16] showed the following: pre, 25 points; post 1,27 points; post 2, 26 points; and post 3, 29 points; $\mathrm{NAP}=$ strong effect. Working memory [16] showed the following: pre, 5 points; post 1,10 points; post 2 ,
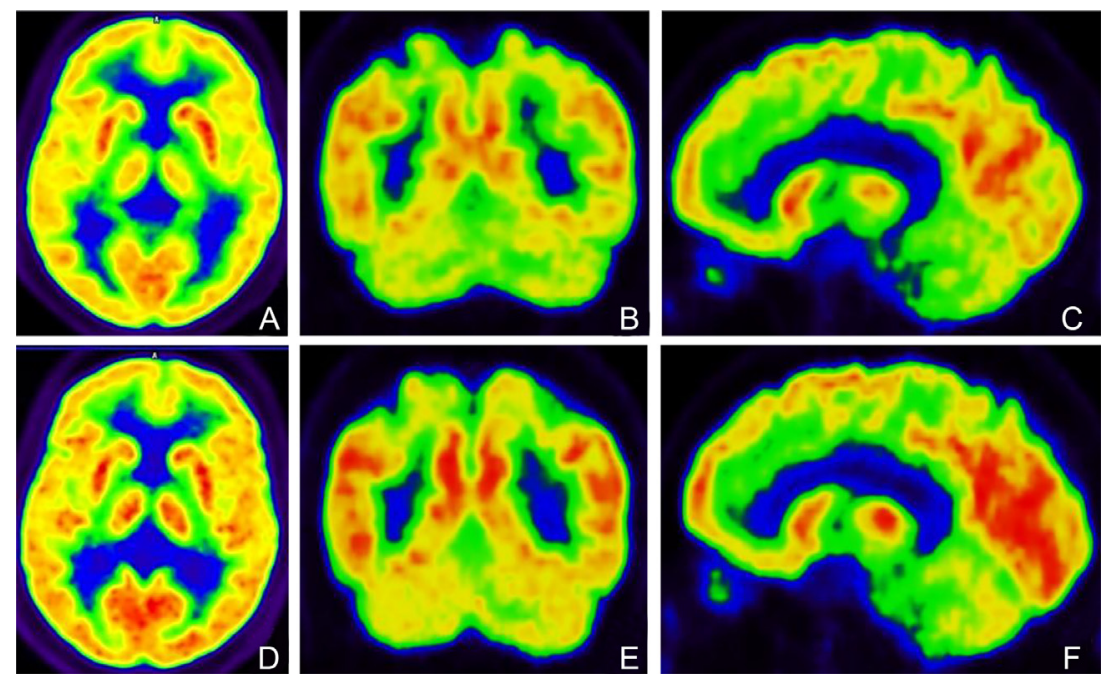

Figure 2. Case 2. 18F-FDG-PET showing metabolic activity prior to cell therapy $(\mathrm{A}-\mathrm{C})$ and at the end of treatment (D-F). A clear increase in cerebral metabolism can be seen at the end of cell therapy. 
11 points; and post 3, 12 points; NAP = strong effect. Study of inhibition [14] showed the following: pre, 47 points; post 1, 54 points; post 2, 49 points; and post 3, 58 points; NAP = strong effect.

In the course of cell therapy, injections of MSCs induced a progressive increase in brain glucose metabolism (Figure 3).

In May 2018, 1 year after the end of the cell therapy, a new assessment was made. At this time, MMSE score [12] was 26, and the patient showed worse scores on verbal short-term memory [15], a decrease of 2 points, but he got the same scores on verbal learning [15] and long-term memory [15]. Furthermore, he presented a better performance on semantic memory [16], working memory [16] and inhibition [14]. On the other hand, at this time, brain glucose metabolism decreased when compared with the 18F-FDG-PET scan obtained at the end of treatment, but the patient maintained the clinical improvement that manifested in May 2017.

\section{Discussion}

These cases are examples of patients in the early stages of $\mathrm{AD}$ who experienced the arrest of clinical deterioration or even clear improvement of their symptoms after being treated using intrathecal injections of autologous MSCs. None of them received any specific medication for disease at the time of receiving cell therapy. In the three cases, improvements were associated with significant neuropsychological improvements and with an increase in cerebral glucose metabolism, studied using $18 \mathrm{~F}-$ FDG-PET.

Cell therapy has been considered to be a possible strategy in $\mathrm{AD}$ through neuroprotection and paracrine actions $[18,19]$, and diverse pre-clinical studies with MSCs have shown benefit in rodent models of AD [20-22]. Moreover, in a clinical trial, umbilical cord blood-derived (hUCB) MSCs were injected into the hippocampus and precuneus of nine patients with mild-to-moderate AD [23]. However, after a review of clinical trials using cell therapy for $\mathrm{AD}$, there is no evidence for clinical usefulness [24].

Intrathecal administration of autologous MSCs is safe and allows for the administration of a high number of cells to the whole brain [10], and our present findings show that this type of cell therapy is associated with a clear increase in cerebral glucose metabolism. It can explain early improvements better than if improvement is attributed to cerebral neuroprotection or neurogenesis.

Due to the limited number of patients in our present study, we can not draw conclusions about the benefit of this type of cell therapy in $\mathrm{AD}$, but it is obvious that the relationship between the increase of cerebral glucose metabolism and the intrathecal administrations of autologous MSCs offers a new line of research in the treatment of this disease, mainly taking into account that an impaired brain glucose metabolism is a commonly recognized early feature of $\mathrm{AD}[25,26]$.

Our present findings should be confirmed in a larger number of patients and multiple unknowns
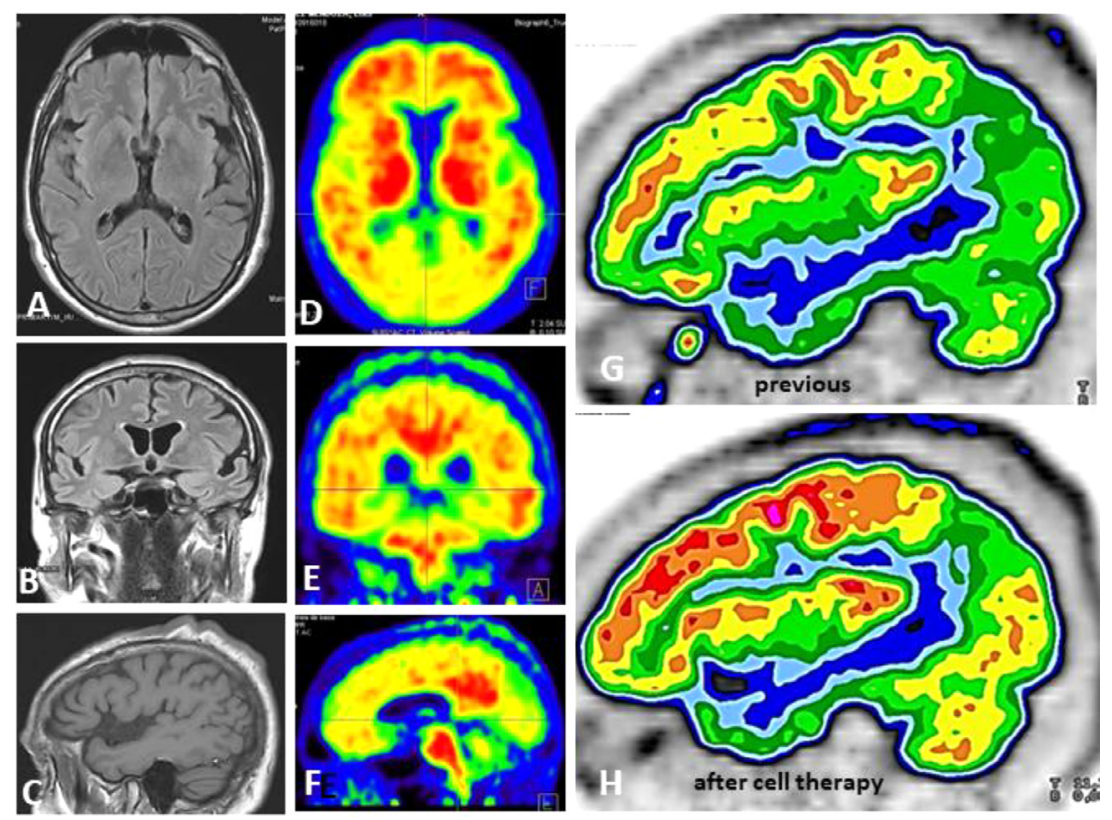

Figure 3. Case 3. (A-C) Magnetic Resonance (MR) showing frontotemporal atrophy, predominantly on the left side. (D-F) $18 \mathrm{~F}-\mathrm{Fluteme}-$ tamol-PET for detection of beta-amyloid neuritic plaques. (G) 18F-FDG-PET performed prior to cell therapy. (H) 18F-FDG-PET at the end of cell therapy. At this time, a strong increase of cerebral glucose metabolism can be seen. 
must be cleared, such as if the effect of the intrathecal administration of MSCs is permanent, or the convenience of a long-term periodic administration of MSCs, in an attempt to maintain a possible therapeutic effect.

\section{Conclusions}

Our present data suggest that intrathecal cell therapy with autologous MSCs increases cerebral glucose metabolism, being associated with neuropsychological improvements in patients experiencing early stages of AD. This type of cell therapy is safe, allowing distribution of donor cells in the whole brain, and its utility for the treatment of $\mathrm{AD}$ and other diseases with cerebral impaired glucose metabolism deserves further studies.

\section{Acknowledgments}

We thank the institutions supporting the development of our cell therapy program, in particular Mapfre and Rafael del Pino Foundations.

Disclosure of interests: The authors have no comercial, proprietary or financial interest in the products or companies described in this article.

\section{References}

[1] Jeonh Y, Cho SS, Park JM, Kang SJ, Lee JS, Kang E, et al. 18F-FDG PET findings in frontotemporal dementia: an SPM analysis of 29 patients. J Nucl Med 2005;46:233-9.

[2] Bert V, Pupi A, Mosconi L. PET/CT in diagnosis of dementia. Ann N Y Acad Sci 2011;1228:81-92.

[3] Ishii K. PET approaches for diagnosis of dementia. AJNR Am J Neuroradiol 2014;35:2030-8.

[4] Mega MS, Cummings JL, O'Connor SM, Dinov ID, Reback E, Felix J, et al. Cognitive and metabolic responses to metrifonate therapy in Alzheimer disease. Neuropsychiatry Neuropsychol Behav Neurol 2001;14:63-8.

[5] Tzimopoulou S, Cunningham VJ, Nichols TE, Searle G, Bird NP, Mistry P, et al. A multi-center randomized proofof-concept clinical trial applying $\left[{ }^{18} \mathrm{~F}\right]$ FDG-PET for evaluation of metabolic therapy with rosiglitazone $\mathrm{XR}$ in mild to moderate Alzheimer's disease. J Alzheimer's Dis 2010;22: 1241-56.

[6] Shimada A, Hashimoto H, Kawabe J, Higashiyama S, Kai Y, Kataoka K, et al. Evaluation of therapeutic response to donepezil by positron emission tomography. Osaka City Med J 2011;57:11-9.

[7] Vaquero J, Zurita M, Bonilla C, Fernández C, Rubio JJ, Mucientes J, et al. Progressive increase in brain glucose metabolism after intrathecal administration of autologous mesenchymal stromal cells in patients with diffuse axonal injury. Cytotherapy 2017;19:88-94.

[8] World Medical Association Declaration of Helsinki. Ethical principles for medical research involving human subjects. JAMA 2000;284:3043-5.

[9] International Conference on Harmonisation Expert Working Group ICH 22 armonized tripartite guideline. Guideline for good clinical practice. 1996. http://www.ich.org/fileadmin/
Public_Web_Site/ICH_Products/Guidelines/Efficacy//E6/ E6_R1_Guideline.pdf.;1996.

[10] Vaquero J, Zurita M, Rico MA, Bonilla C, Aguayo C, Fernández C, et al. Repeated subarachnoid administrations of autologous mesenchymal stromal cells supported in autologous plasma improve quality of life in patients suffering incomplete spinal cord injury. Cytotherapy 2017;19:349-59.

[11] Vaquero J, Zurita M, Rico MA, Bonilla C, Aguayo C, Montilla J, et al. An approach to personalized cell therapy in chronic complete paraplegia: The Puerta de Hierro phase I/II clinical trial. Cytotherapy 2016;18:1024-35.

[12] Folstein MF, Folstein SE, McHugh PR. Mini-mental status. A practical method for grading the cognitive state of patients for the clinician. J Psychiatr Res 1975;12:189-98.

[13] Rey A. L'examen psychologique dans les cas d'encephalopathie traumatique (Les problems). Arch Psychol 1941;28:215-85.

[14] Stroop JR. Studies of interference in serial verbal reactions. J Exp Psycho 1935; 18:643-62.

[15] Sales-Galán A, Meléndez-Moral JC, Mayordomo-Rodríguez $\mathrm{T}$. Using a cognitive plasticity measure to detect mild cognitive impairment. Arch Clin Neuropsychol 2013;28:763-70.

[16] Wechsler D. Wechsler Memory Scale-Third Edition manual. San Antonio, TX: The Psychological Corporation; 1997.

[17] Parker RI, Vannest KJ. An Improved effect size for single case research: NonOverlap of All Pairs (NAP). Behav Ther 2009;40:357-67.

[18] Amemori T, Jendelova P, Ruzicka J, Urdzikova LM, Sykova E. Alzheimer's disease: Mechanism and approach cell therapy. Int J Mol Sci 2015;16:26417-51.

[19] Duncan T, Valenzuela M. Alzheimer's disease, dementia, and stem cell therapy. Stem Cell Res Ther 2017;8:111.

[20] Bae JS, Jin HK, Lee JK, Richardson JC, Carter JE. Bone marrow-derived mesenchymal stem cells contribute to the reduction of amyloid- $\beta$ deposits and the improvement of synaptic transmission in a mouse model of pre-dementia Alzheimer's disease. Curr Alzheimer Res 2013;10:524-31.

[21] Oh SH, Kim HN, Park HJ, Shin JY, Lee PH. Mesenchymal stem cell increase hippocampal neurogenesis and neuronal differentiation by enhancing the Wnt signaling pathway in an Alzheimer's disease model. Cell Transplant 2015;24:1097-109.

[22] Shin JY, Park HJ, Kim HN, Oh SH, Bae JS, Ha HJ, Lee PH. Mesenchymal stem cells enhance autophagy and increase $\beta$-amyloid clearance in Alzheimer disease models. Autophagy 2014;10:32-44.

[23] Kim HJ, Seo SW, Chang JW, Lee JI, Kim CH, Chin J, et al. Stereotactic brain injection of human umbilical cord blood mesenchymal stem cells in patients with Alzheimer's disease dementia: A phase 1 clinical trial. Alzheimer's Dement (NY) 2015;1:95-102.

[24] Kang JM, Yeon BK, Cho SJ, Suh YH. Stem cell therapy or Alzheimer's disease: A review of recent clinical trials. J Alzheimer's Dis 2016;54:879-89.

[25] Calsolaro V, Edison P. Alterations in glucose metabolism in Alzheimer's disease. Recent Pat Endocr Metab Immune Drug Discov 2016;10:31-9.

[26] Chen Z, Zhong C. Decoding Alzheimer's disease from perturbed cerebral glucose metabolism: implications for diagnostic and therapeutic strategies. Progr Neurobiol 2013;108: 21-43.

\section{Supplementary materials}

Supplementary material associated with this article can be found in the online version at doi:10.1016/j. jcyt.2019.02.009. 